\title{
EDITORIAL
}

\section{HACIA UN MODELO DE COOPERACIÓN Y ARMONIZACIÓN EN EL CAMPO DE LA SALUD PÚBLICA EN ESPAÑA}

\author{
Jose M. ${ }^{a}$ Martín Moreno
}

Director General de Salud Pública. Ministerio de Sanidad y Consumo

La salud, entendida como un estado de equilibrio, adaptación y bienestar (físico, mental y social), expresa un ideal que se incluye entre los objetivos esenciales de toda política pública coherente.

La Salud Pública se puede identificar con el conjunto de esfuerzos organizados de la sociedad de acuerdo con una misión esencial: prevenir problemas de salud y fomentar la salud de la comunidad. Es primordial en esa labor la convergencia en el trabajo de personas que idealmente tienen un alto grado de especialización, aunque provengan de diferentes perfiles profesionales de partida. Entre las dimensiones cardinales de la Salud Pública, destacan: su concepción filosófica basada en la equidad; las inevitables implicaciones políticas que conlleva (desde las enfermedades infecciosas a los riesgos ambientales, pasando por las enfermedades crónicas, la organización de la atención sanitaria, los riesgos laborales, y la promoción de la salud en las personas mayores,...); su amplio ámbito de actuación; el carácter multidisciplinario propio de su naturaleza; y el impacto e interrelación multisectorial (trabajo, ambiente, educación, alimentación...) que subyace en los fenómenos que afectan a la salud ${ }^{1}$. Sintetizando la esencia del concepto, la Salud Pública incluye tanto la salud del público como las dimensiones colectivas de la salud²

En España, hablando del terreno de la Salud Pública, vivimos un momento especialmente interesante por lo que ofrece de opor- tunidades, aunque ello vaya a su vez acompañado de complejos retos. Actualmente, tal y como es admitido desde la mayoría de los diferentes sectores, incluyendo el sistema sanitario, la dimensión de integración y armonización es fundamental para la Salud Pública $^{3}$ y, por tanto, son cruciales los instrumentos de cooperación y colaboración con las Comunidades Autónomas (CCAA) y las Administraciones locales, todo ello en un marco europeo e internacional que demanda cada vez mayor cohesión. Mucho se ha hecho, pero queda aún mucho por hacer ${ }^{4}$. Así, cuando nos encontramos con problemas estructurales se da la circunstancia de que, al igual que en otras dimensiones de la vida, llegamos a tener la sensación de vivir en un mundo de inercias, en el que las cosas se hacen porque ya venían haciéndose de esa forma... pudiendo existir la tentación de concluir que hay muchos principios sin continuidades y muchas continuidades sin principios $^{5}$. Pues bien, para poner las cosas en su contexto, es importante revisar, aunque sólo sea de forma resumida, el escenario normativo y los orígenes recientes de los que partimos.

\section{CONTEXTO NORMATIVO DE PARTIDA}

Teniendo en cuenta que la Constitución Española de 27 de diciembre de 1978 (este número de la revista coincide con su 24 aniversario) es la norma legal de más alto ran- 
go, empezaremos citando su artículo 43, en el que la Carta Magna reconoció el derecho a la protección de la salud, estableciendo que compete a los poderes públicos organizar y tutelar la salud pública a través de medidas preventivas y de las prestaciones y servicios necesarios. Derecho que también se contempla en otros artículos constitucionales.

Asimismo, estableció en su artículo 148.1.21. que las Comunidades Autónomas podrán asumir competencias en materia de Sanidad e higiene, mientras en su artículo 149.1.16 señalaba que el Estado tiene competencia exclusiva sobre sanidad exterior, bases y coordinación general de la sanidad y legislación sobre productos farmacéuticos.

Las actividades de Sanidad Exterior, con más de cien años de existencia formal (Reglamento Orgánico de Sanidad Exterior, de 27 de octubre de 1899), tienen por objeto la vigilancia y el control de los riesgos de salud derivados de la importación, exportación y tránsito de mercancías, y del tráfico internacional de viajeros (artículo 38.2 de la Ley 14/1986, General de Sanidad). Desde 1997, los Servicios Periféricos de Sanidad dependientes de la Administración del Estado se encuentran estructurados a través del Real Decreto 1330/1997, de integración de servicios periféricos y de estructura de las Delegaciones del Gobierno, por lo que las Unidades Periféricas de Sanidad disponen de una dependencia orgánica del Ministerio de Administraciones Públicas mientras existe una dependencia y una dependencia funcional del Ministerio de Sanidad y Consumo.

En cuanto al contexto que establece las competencias del Ministerio de Sanidad y Consumo en materia de Sanidad Ambiental, se enmarca en:

- La Constitución Española, que en su artículo 45 recoge las actuaciones en materia de Sanidad Ambiental.

- La Ley 14/1986, de 25 de abril, General de Sanidad (artículos 6.3, 8.1, 8.2, 18.6,
$18.11,19,21,22,23-28,39,40.1,40.2$ y disposición adicional segunda)

- La legislación y reglamentación técnico-sanitaria específica sobre: biocidas, plaguicidas, preparados peligrosos, notificación de sustancias nuevas, limitación de uso de sustancias y preparados peligrosos, protección radiológica del paciente, efectos para la salud de los campos electromagnéticos, control de la calidad del agua de consumo humano y control de la calidad del agua de baño.

Finalmente, señalar que en materia de Salud Laboral, la Ley 31/1995 de Prevención de Riesgos Laborales ha venido a retomar el Capítulo IV de la Ley General de Sanidad «De la salud laboral», a la vez que ha definido nuevas competencias para las administraciones sanitarias en este ámbito.

Por otro lado, la Ley 14/1986, de 25 de abril, General de Sanidad, dio respuesta a dos requerimientos constitucionales de máxima importancia, incluyendo el ya mencionado reconocimiento del derecho a la protección a la salud (artículo 43 y concordantes de la Constitución), y la incidencia en nuestra organización sanitaria de las previsiones del título VIII, con la definición del papel de las Comunidades Autónomas, sobre las que se articula el Sistema Nacional de Salud (SNS).

Pues bien, la Constitución contempló la posibilidad y los Estatutos de Autonomía la ejercitaron, de que las competencias sobre salud pública fueran transferidas a las CCAA. Los distintos Reales Decretos de transferencia materializaron la decisión.

Recordemos que tras la aprobación de nuestra Constitución se pusieron en marcha importantes transformaciones del sistema de salud, los cuales se desarrollaron sobre todo con las reformas sanitarias y de la administración pública durante los años ochenta. Éstas produjeron, entre otras consecuencias, una importante descentralización hacia las Comunidades Autónomas. 
Dicha descentralización, con el traspaso de competencias correspondiente, supuso un cambio radical respecto a la situación precedente, de forma que en el subsistema de salud pública la situación es prácticamente la propia de una estructura cuasi federal.

\section{ALGUNAS CONSIDERACIONES SOBRE EL ÁMBITO DE LA SALUD PÚBLICA DESDE LA PERSPECTIVA DEL MINISTERIO DE SANIDAD Y CONSUMO}

Lo anteriormente expuesto ha comportado una importante redefinición de funciones para los servicios de la Administración Central del Estado.

Al revisar los indicadores básicos de salud, podemos constatar que España goza de una situación, que en muchos casos puede considerarse como privilegiada en el mundo ${ }^{6,7}$. La elevada esperanza de vida de las ciudadanas y ciudadanos españoles y, por citar otro indicador, la baja mortalidad infantil, son elementos de los que podemos estar orgullosos. Al mismo tiempo, en el contexto de los estados miembro de la Organización Mundial de la Salud, nuestro sistema sanitario es ciertamente destacable ${ }^{8}$. Sin embargo, ello no nos puede llevar a la autocomplacencia, dado que la incidencia y prevalencia de las enfermedades cardiovasculares, del cáncer, de los accidentes, y de ciertas enfermedades transmisibles como el sida y otras emergentes y reemergentes, junto con la aparición de nuevos problemas sanitarios, siguen exigiendo una atención preferente a la política de promoción de la salud y de prevención de enfermedades, así como a una reorientación de los cuidados basada en la profundización de la solidaridad que a su vez promueva salud.

Por otro lado, nuestro carácter de Estado Miembro de la UE exige el control higiénico-sanitario de puertos, aeropuertos y medios de transporte internacional, el seguimiento higiénico-sanitario de mercancías en régimen de importación y exportación. Además, hemos de garantizar el cumplimiento en España de la numerosa legislación europea relacionada con los factores ambientales que determinan nuestra salud. Para ello es necesario fortalecer los mecanismos de coordinación y cooperación con las CCAA en materia de protección de la salud. La exposición a agentes contaminantes y factores de riesgo afecta a todos los ciudadanos/as independientemente de donde vivan, pues no respetan fronteras y tienen un impacto demostrado en la incidencia y prevalencia de numerosas enfermedades (respiratorias, cardiovasculares, cáncer, intoxicaciones, etcétera).

Además, algunos de los retos que podríamos englobar como de equidad en Salud Pública, han podido verse intensificados una vez concluido el proceso de transferencias en materia de salud pública a las Comunidades Autónomas. Esta circunstancia, junto con algunas otras que rodean al problema (ausencia de evidencias científicas estables, nuevos fenómenos o situaciones intensificadas como la de la inmigración...) aconsejan el desarrollo de planes de actuación para que su puesta en marcha se haga, además de a partir de la legitimidad de los propios Servicios de las CCAA, de una manera global para el conjunto del Estado, mediante una planificación y coordinación activa del Ministerio de Sanidad y Consumo.

Por todo lo anterior, el Ministerio de Sanidad y Consumo ha hecho explícito su compromiso de promover y mejorar la salud, prevenir las enfermedades y garantizar la mejor coordinación en la lucha contra amenazas potenciales para la salud, todo ello con el objeto de reducir la morbilidad, la discapacidad y la mortalidad prematura evitable. Para contribuir al bienestar de los españoles, el Sistema Nacional de Salud debe atender de manera coordinada y coherente las preocupaciones de su población acerca de los riesgos sanitarios y sus expectativas de un nivel elevado de protección de la salud. Por ello todas las actividades del Ministerio de Sanidad y Consumo deben darse a conocer y ser transparentes, así como permi- 
tir la consulta y participación equilibradas de todos los agentes interesados, con el propósito de fomentar la mejora de los conocimientos y de los flujos de comunicación, haciendo así posible una participación más amplia de las personas en las decisiones que afectan a su salud.

En el momento actual, y en función del Real Decreto 840/2002, de 2 de agosto, por el que se modifica y desarrolla la estructura orgánica básica del Ministerio de Sanidad y Consumo, la Dirección General de Salud Pública es el órgano que asume las funciones relativas a la información epidemiológica, la promoción de la salud y la prevención de las enfermedades, la sanidad exterior, la salud laboral, el control sanitario del medio ambiente y los requisitos higiénico-sanitarios de los productos de uso y consumo humano, así como la elaboración de la normativa en estas materias. Asimismo, le corresponde la determinación de los criterios que permitan establecer la posición española ante la Unión Europea y en los foros internacionales en las materias directamente relacionadas con la Salud Pública, sin perjuicio de las competencias de otros Departamentos Ministeriales.

Entre los PRINCIPIOS RECTORES que inspiran nuestra estrategia destacan:

a) Coordinación institucional y participación

- Coordinación de las políticas de salud pública y multisectorialidad. La multisectorialidad, que caracteriza a las actuaciones realizadas en el ámbito de la salud pública, determina la necesidad de que la administración sanitaria establezca alianzas estratégicas con el sistema educativo, los servicios sociales, los representantes de los trabajadores..., todo ello en colaboración activa con las administraciones sanitarias autonómicas y locales.

- Participación ciudadana. Contempla la acción sinérgica entre instituciones de las administraciones públicas, privadas y organizaciones no gubernamentales para la planificación, implementación y evaluación de estrategias y programas.

- Interdisciplinariedad y profesionalización. Interacción de profesionales especializados provenientes de distintos ámbitos.

- Articulación en el SNS de las políticas europeas y compromisos internacionales.

b) Evaluación sistemática de programas: aplicación de estrategias de eficacia demostrada ${ }^{9}$.

- Principio reducción de daños. Entendido como un elemento más de nuestra política preventiva. Las medidas pragmáticas orientadas a reducir la morbi-mortalidad y mejorar la calidad de vida han demostrado reiteradamente ser más efectivas que las intervenciones maximalistas, no sólo para reducir la transmisión de agentes infecciosos (por ejemplo el VIH) y/o de otros hábitos o prácticas nocivas, sino para crear un entorno favorable para el acceso a los servicios sanitarios y sociales y la reducción de la marginalidad. En esta misma línea de seleccionar las estrategias con criterios de efectividad destacan también la educación entre iguales, la participación de mediadores en los programas, el trabajo de acercamiento, el enfoque específico de género y el uso de los medios de comunicación.

- Vinculación con estrategias afines que promuevan salud.

c) Equidad

- Derechos humanos, tolerancia y solidaridad. La equidad debe impregnar desde la etapa de planificación todas las actividades preventivas, asistenciales, de investigación y de vigilancia epidemiológica que se realicen. Los diferentes estilos de vida son parte esencial de 
una sociedad pluralista y abierta, y el respeto y la solidaridad, elementos básicos para que cada persona y la comunidad a la que pertenecen se sientan plenamente responsables y participativos.

- Igualdad de oportunidades y no discriminación. El acceso equitativo a las medidas de prevención, al tratamiento y al apoyo social constituye uno de los elementos fundamentales del sistema sanitario español y requiere medidas positivas para aquellas personas o grupos que por su situación de exclusión social o diferencias culturales se encuentran en situación de vulnerabilidad.

- Reducción de la vulnerabilidad, reconociendo los determinantes políticos, económicos, sociales, culturales, ambientales, conductuales y biológicos de la salud a la hora de diseñar las intervenciones.

\section{LA NECESIDAD DE LA COORDINACIÓN A TRAVÉS DE LA MEJOR COOPERACIÓN Y ARMONIZACIÓN DE ACCIONES EN SALUD PÚBLICA}

En el marco descentralizado previamente descrito, el ejercicio de la coordinación general del Sistema Nacional de Salud (SNS), en lo que atañe a la Salud Pública, es esencial por cuanto los efectos de los problemas trascienden normalmente el ámbito regional o autonómico.

Por ello, y con el objetivo último de articular nuestro papel efectivo en el contexto de la interterritorialidad y a su vez la internacionalidad, la interdisciplinariedad en el abordaje de la disciplina y la intersectorialidad en su puesta en práctica, nos hemos propuesto, desde la actual Dirección General de Salud Pública del Ministerio de Sanidad y Consumo, un plan de trabajo que realmente posibilite un funcionamiento coordinado, armonizado y de sinérgica cooperación, que esté preparado para responder ante situaciones de alarma para la salud pública en España, y para el desarrollo de planes integrales de prevención y control de las enfermedades, y de protección y promoción de la salud de los ciudadanos. Nuestra estrategia intentará la mayor coherencia con el Programa Europeo de Acción Comunitario en el ámbito de la Salud Pública 2003-2008 (Decisión N. ${ }^{\circ} 1786 / 2002 / \mathrm{CE}$ del Parlamento Europeo y del Consejo, publicada en el Diario Oficial de las Comunidades Europeas de 9 de octubre de 2002). La filosofía que inspira nuestras acciones es la de intentar recoger todo lo positivo de la labor que ya se viene realizando, mientras mejoramos aquellos procedimientos necesarios para lograr un enfoque coherente y eficaz de las cuestiones relacionadas con la salud en el conjunto de los diferentes ámbitos que cubren las políticas de las administraciones públicas.

En definitiva, para desarrollar adecuadamente sus funciones, la salud pública necesita mantener una capacidad de actuación. La actividad de salud pública no puede estar poniéndose en marcha y parándose de forma intermitente a medida que se identifican problemas y situaciones nuevas. Es preciso que mantenga su capacidad de actuar de forma permanente para que cumpla sus exigencias con la salud de la sociedad.

Para ello se precisa un liderazgo efectivo, voluntad política, base científica sólida y contrastada, profesionales competentes, herramientas para monitorizar el estado de salud de la población orientadas a la acción, organización y estructuras adecuadas, recursos humanos y físicos adecuados al proyecto, y un sistema normativo que permita su acción eficaz.

Lo deseable es el desarrollo de planes de actuación para la protección, promoción de la salud y prevención de las enfermedades más prevalentes, de una manera global para el conjunto del Estado, respetando el papel destacado de las Comunidades Autónomas, y sin olvidar a los Municipios y Provincias, pero 
contando también con la participación activa del Ministerio de Sanidad y Consumo.

Para todo lo anterior, nos proponemos poner en marcha un Plan de Cooperación y Armonización en el ámbito de la Salud Pública en España, para la vertebración de las acciones de las Administraciones Públicas en este ámbito. Desde su concepción, este Plan está ideado para realizarse en estrecha coordinación con las CCAA y buscamos que incluya, desde una visión integral de la Salud Pública, la coordinación de sistemas de información epidemiológica y de salud pública, de respuesta ante situaciones de emergencia o alarma sanitaria, de promoción y protección de la salud de la población española, y de prevención de las enfermedades más prevalentes. En estos momentos, se está trabajando en la elaboración detallada de este Plan. Entre los objetivos que podemos avanzar, a modo de propuestas iniciales, están los siguientes:

- Potenciar la coordinación estatal en este ámbito, a través de la Comisión de Salud Pública del Consejo Interterritorial del Sistema Nacional de Salud, y de la integración de la Salud Pública en la Política de Coordinación del Sistema Nacional de Salud, contando con todas las administraciones públicas (local, autonómica y estatal) y con la mayor participación ciudadana.

- Fomentar la coordinación interdepartamental de la Salud Pública, con el objeto de garantizar la vinculación, cohesión y compromiso de las distintas áreas del gobierno implicadas en un mismo problema de salud.

- Establecer un Plan de Respuesta ante situaciones de Alarma para la Salud Pública en colaboración con las CCAA (incluyendo temas desgraciadamente candentes como el bioterrorismo).

- Establecer un Plan de Calidad en Sanidad Exterior, que incluirá la creación de una Carta de Servicios de Sanidad
Exterior, el establecimiento de indicadores de gestión y de actividad, y la designación de objetivos y compromisos de cara al administrado.

- A partir de la Comisión de Salud Pública del Consejo Interterritorial del Sistema Nacional de Salud, organizar Foros de Debate sobre la Salud Pública en España, en los que se espera tener en cuenta la voz de los diferentes agentes sociales, las sociedades científicas y las asociaciones profesionales, ello para asegurar que todo lo anteriormente expuesto se haga a partir del conocimiento científico y pragmático y el máximo consenso.

Entre todos, combinando razón y pasión, podremos llegar a superar los diferentes retos y seguir avanzando en el mundo de la Salud Pública.

\section{BIBLIOGRAFÍA}

1. Martín-Moreno JM. Salud Pública en Europa: Papel de las Escuelas de Salud Pública y Reflexiones Globales. En: Organización Panamericana de la Salud. Educación en salud pública. Nuevas perspectivas para las Américas. Washington DC: OPS; 2001: 224-34.

2. Guerra de Macedo C. La Salud Pública en las Américas: Documento conceptual y operacional. En: Organización Panamericana de la Salud. Educación en salud pública. Nuevas perspectivas para las Américas. Washington DC: OPS; 2001: 3-16.

3. Villalbí JR, Aboal XL, González-Alonso J. Los servicios de salud pública: progresos y problemas prioritarios. En: JM Cabasés, JR Villalbí, C Aibar (eds.) Informe SESPAS 2002. Valencia: Escuela Valenciana de Estudios para la Salud; 2002: 545-64.

4. Segura A, Villabí JR, Mata E, De la Puente ML, Ramis-Juan O, Tresserras R. Las estructuras de salud pública en España: un panorama cambiante. Gac Sanit 1999; 13: 218-25.

5. Martín-Moreno JM. «El futuro de las Escuelas de Salud Pública: Principios, continuidades y esperanzas». En: Sepúlveda J eds. Retos de la Cooperación Internacional en el Desarrollo del Capital Hu- 
mano. Cuernavaca (México): Instituto Nacional de Salud; 2002

6. Regidor E, Gutiérrez-Fisac JL. Indicadores de Salud. Cuarta evaluación en España del Programa Regional Europeo Salud para Todos. Madrid: Ministerio de Sanidad y Consumo; 1999.

7. EUROSTAT. Datos claves sobre la salud. Luxemburgo: Eurostat; 2000
8. Organización Mundial de la Salud. Informe sobre la salud en el mundo 2000. Mejorar el desempeño de los sistemas de salud. Ginebra: Organización Mundial de la Salud; 2000.

9. Cid-Ruzafa J, Rodríguez-Artalejo F, Martín-Moreno JM. ¿Hacia una Salud Pública Basada en la Evidencia? Med Clín (Barc) 1999; 112 (Supl. 1): 106-110. 